\title{
Controlling the Meso-scale Assembly of CNTs/PBI Interlayers for Toughening of Thermoplastic Composites
}

\author{
Kaan Yildiz ${ }^{1}$ \\ Faculty of Aeronautics and Astronautics, Istanbul Technical University, Istanbul 34469, Turkey \\ Aerospace Research Center, Istanbul Technical University, Istanbul 34469, Turkey
}

Beyza Bozali ${ }^{2}$

Faculty of Textile Technologies and Design, Istanbul Technical University, Istanbul 34437, Turkey Aerospace Research Center, Istanbul Technical University, Istanbul 34469, Turkey

Hulya Cebeci ${ }^{3}$

Faculty of Aeronautics and Astronautics, Istanbul Technical University, Istanbul 34469, Turkey Aerospace Research Center, Istanbul Technical University, Istanbul 34469, Turkey

Elif Ozden-Yenigun ${ }^{4}$

School of Design, Textile, Royal College of Art, SW7 2EU, London, United Kingdom

\begin{abstract}
Utilizing the grown carbon nanotubes (CNTs) on nanofibers having high surface area provides an ability to tailor mechanical, electrical and thermal performance in various applications such as lithium-sulfur batteries, laminated composites, and supercapacitors. In this study, growth of CNTs on curved polymeric nanofibrous substrates is conducted to address single-step CNTs synthesis on polybenzimidazole (PBI) nanofibers as an interlayer, which benefits from the synergetic effect of PBI nanofiber network and stiff and conductive CNTs. The growth of radially oriented CNTs on PBI nanofibrous substrates at $600{ }^{\circ} \mathrm{C}$ has successfully achieved by chemical vapor deposition method (CVD). Then, these interlayers are used for toughening in CF/PEEK laminated composites. The integration of CNTs-PBI interlayers into CF/PEEK laminated composites presented promising results under flexural loadings.
\end{abstract}

\section{Introduction}

Thermoplastic resin-based laminated composites with remolding/reshaping ability, recyclability, and multifunctionality, if combined with reinforcement agents, have drawn attention in the composite industry. However, incorporation problems of reinforcement agents such as CNTs into thermoplastic matrices have been challenging due to the difficulties in processability at elevated temperatures and poor dispersion, which leads to weak interfacial bonding with the polymer matrix $[1,2]$. The implementation of CNTs as a reinforcing agent to interlaminar regions could be achieved by different approaches, including placement of a strong buckypaper as an interleave, nano-stitching of CNTs, coating of fibers with CNTs-containing sizes, the growth of CNTs onto the fiber, etc. [3-6]. This study aims

\footnotetext{
${ }^{1}$ Postdoctoral Researcher, Department of Aeronautical and Astronautical Engineering

${ }^{2} \mathrm{PhD}$ Student, Department of Textile Engineering

${ }^{3}$ Associate Professor, Department of Aeronautical Engineering, AIAA member

${ }^{4}$ Senior Tutor, School of Design, Textiles
} 
to investigate the potential improvements in the mechanical performance of laminated composites as a result of adding CNTs to the interlayers.

Several studies focused on the enhancement of mechanical properties of composites by the addition of CNTs to structures. Kim et al. reported that the flexural strength of alumina matrix composites reinforced with CNTs was enhanced with the increasing CNT content and reached a maximum at 1.5 vol.\% CNTs. Even though this increase can be explained with the strengthening mechanism of CNTs, further increases in CNTs fraction led to a decline in the flexural strength [7]. Similar behavior was reported by Cho et al. who observed an increase in the flexural strength with up to 1 vol.\% multi-walled carbon nanotubes (MWCNTs) addition. However, the flexural strength was found to be decreasing with increased MWCNTs content. This behavior was associated with the formation of voids, the agglomeration and poor dispersion of CNTs in the polyphenylene sulfide (PPS) matrix [8]. In another study by Kim et al., it was observed that addition of low-content CNTs $(0.3 \mathrm{wt} \%)$ into resin presented a little effect on tensile properties, while flexural modulus, strength, and percent strain to break were improved by $11.6 \%, 18.0 \%$, and $11.4 \%$, respectively, in carbon fiber (CF) reinforced epoxy composites [9]. Liang et al. conducted experimental studies to evaluate how the impact and flexural properties of polypropylene (PP) composites vary with the introduction of MWCNTs. The main findings of their study include enhancements in the flexural modulus and stiffness due to the presence of MWCNTs, regardless of their diameter and average length-to-diameter ratio. The improvements in the flexural stiffness were attributed to promoted crystallization of the PP matrix as a result of the heterogeneous nucleation effect of the MWCNTs [10].

To date, studies focusing on the mechanical properties of CNT-reinforced materials majorly investigates the improvement of mechanical properties of thermoset-based composite structures [11-13], while the reports on CNTreinforced thermoplastic-based composites are quite rare. Diez-Pascual et al. examined the mechanical performance of CNT-integrated thermoplastic laminates and reported increases in storage modulus ( $\left.E^{\prime}\right)$ and glass transition temperature $\left(\mathrm{T}_{\mathrm{g}}\right)$ with the integration of arc-grown single-walled carbon nanotubes (SWCNTs) and laser-grown SWCNTs. The report also showed that the use of compatibilizer (Polysulphone-PS) hindered the SWCNTs agglomeration formation at the interface region, explaining the higher glass transition temperatures [14]. Sandler et al. also observed the increment in the $\mathrm{T}_{\mathrm{g}}$ since the presence of CNTs causes a decrease in polymer chain motions [15]. In another study, $30 \%$ enhancement in the storage modulus was shown to be possible by the addition of MWCNTs into the Polyamide 6 (PA 6)/Glass Fiber (GF) composites because of the interconnecting effect of MWCNTs within the thermoplastic matrix. Another outcome of the interconnecting effect was reported to be greater $\mathrm{T}_{\mathrm{g}}$, measured from the temperature curves of MWCNT-reinforced composites [16]. Jin et al. also stated a similar, increasing behavior for $\mathrm{E}^{\prime}$ and $\mathrm{T}_{\mathrm{g}}$ by the addition of CNTs, showing a stiffening effect at high temperatures [17]. Teng et al. studied and optimized the effect of MWCNT content on the mechanical properties of PP composites. The study reports that up to a certain level, storage modulus increased by the addition of MWCNTs, but aggregation occurs at high MWCNTs contents, which results in a decrease in the storage modulus [18].

The growth procedure of CNTs can have a significant influence on the mechanical properties of the CNT-reinforced composite structures. Lv et al. studied the effect of growth time on CNTs orientation over CFs. They reported that up to 120 min., aligned growth of CNTs were successfully achieved due to the steric interaction of nanotubes while suggesting caution with catalyst density of CNTs as lower density values might result in uncontrolled growth of CNTs, leading to entanglement over fibers [19]. Agnihotri et al. studied the effect of CNTs growth times on the storage modulus of CNT-coated CF/Polyester composites and reported that the $\mathrm{E}^{\prime}$ values were increased gradually by increasing the growth time from 5 to 20 minutes. The reason for this increase was attributed to the larger contact area causing mechanical interlocking between the polymer matrix and the fiber. Beyond 20 minutes of growth time, mechanical interlocking was found to be infeasible due to higher CNTs density [20].

Nowadays, it is still a big challenge to effectively produce CNT-reinforced thermoplastic laminates due to their high processing temperatures and viscosity. To effectively produce CNT-reinforced interleaves with a high CNT quality, it is required to propose an alternative method to overcome these implementation problems. In this paper, a novel method to synthesize radially aligned CNTs directly onto PBI nanofiber substrates by CVD method is proposed, which benefits from the synergetic effect of PBI nanofibers and CNTs. Additionally, direct synthesis of CNTs onto polymeric nanofibers has the potential of their implementation as interleaves without tarnishing the quality of the CNTs, as delamination, collection, dispersion, and distribution steps would have been avoided, resulting in a single-step approach. The incorporation of these produced CNTs-PBI nanofibrous substrates as interlayers into thermoplastic laminates is investigated in this work. The laminated composite structures in which CF/PEEK prepregs were used as the outermost layers and Polyethersulphone (PES) matrix was interlayered with CNTs-PBI nanofibers. To characterize the effect of PBI and CNTs-PBI interlayers on the flexural stiffness of the laminated composites, the stiffness of the laminated composites is characterized by a universal test machine and the results are presented herein. 


\section{Experimental Procedure}

Similar to our previous work [21], PBI nanofibers were produced by dissolving PBI solution (PBI Products, 26.2 wt.\%) in N-dimethylacetamide (DMAc) solvent to prepare a $20 \mathrm{wt} . \%$ solution, followed by an electrospinning process (Argeteknolab). 1 and $10 \mathrm{mM}$ catalyst solutions $\left(\mathrm{Fe}\left(\mathrm{NO}_{3}\right)_{3} \cdot 9 \mathrm{H}_{2} \mathrm{O}(404.0 \mathrm{~g} / \mathrm{molar}\right.$, Emir Kimya) and iso-proponal) (Sigma Aldrich)) were prepared to deposit $\mathrm{Fe}^{3+}$ ions onto nanofibers which acted as a catalyst for the CNT growth. Then, nanofibers were kept in an oven at $50^{\circ} \mathrm{C}$ for 5 hours.

CNT synthesis on PBI nanofibers was achieved by the CVD method, the parameters of which were optimized for uniform conformance and well-distribution of CNTs. The growth temperature was determined as $600{ }^{\circ} \mathrm{C}$ since a significant degradation, which takes place around $780{ }^{\circ} \mathrm{C}$, was not apparent, revealed by previously conducted thermogravimetric analyses. The thermal degradation behavior of neat PBI nanofibers is shown in Fig. 1, revealing the significant weight loss around $780^{\circ} \mathrm{C}$

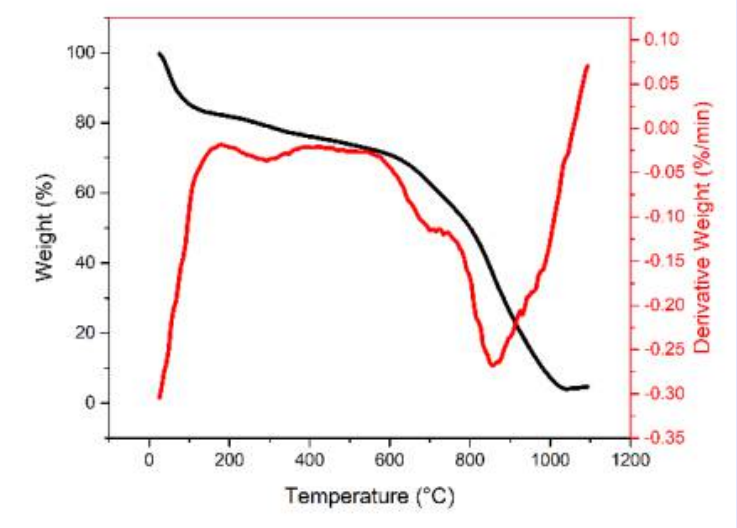

Fig. 1 Thermogravimetric analysis of neat PBI nanofibers

The optimization of the CNT growth procedure was addressed in our previous study [22], accomplished by conducting a set of experiments in which parameters such as catalyst molarity, nucleation time, and growth time were varied to achieve uniform conformance and well-distribution of CNTs onto PBI. The catalyst molarity was selected to be 1 and $10 \mathrm{mM}$ while the nucleation and growth times were chosen as 10 and $15 \mathrm{~min}$, resulting in 8 different growth protocols. The optimum CVD parameters were found to be catalyst molarity of $10 \mathrm{mM}$, nucleation time of 15 min and growth time of 10 min.

The growth of CNTs onto PBI nanofibers was then achieved with the optimized parameters, and the produced CNTs-PBI nanofibrous substrates were used as interleaves in the laminated composites without any surface modification. Before their integration to laminated composite structures as interlayers, morphological characterizations were performed. Scanning Electron Microscopy (SEM) and Transmission Electron Microscopy (TEM) were used to investigate the morphology of the produced CNTs-PBI nanofibers. SEM analysis was conducted by a QUANTA FEG SEM under various magnifications operated at $15 \mathrm{kV}$. For TEM analyses, CNTs-PBI nanofibrous substrates were initially dissolved in ethanol solvent using an ultrasonic cleaner for 10 minutes. Then, the resulting smaller pieces were placed onto grid surfaces (EMS CF200-Cu) under an optical microscope. The prepared grids were investigated carefully by a JEOL 2100 HRTEM operated at $200 \mathrm{kV}$ in which the imaging of individual CNTs was targeted. Furthermore, in order to verify the presence and evaluate the quality of CNTs, Raman spectroscopy was conducted by Renishaw inVia reflex microscopy with an excitation energy of $2.32 \mathrm{eV}$ and acquisition in the range of $100-3000 \mathrm{~cm}^{-1}$.

Following the morphological characterizations of CNTs-PBI nanofibers, among various high-performance thermoplastic polymers including PES, Polysulphone (PS), and Polyetherimide (PEI), PES polymer was found to be yielding good compatibility with the PBI substrates according to the calculated Hildebrand solubility parameters, after a short molecular dynamics simulation using the software of Materials Studio [23]. Hildebrand solubility parameters, following the protocol in [23] were calculated for each monomer of the selected thermoplastic polymers using Equation 1 as follows: 


$$
\delta=\left(\frac{\Delta \mathrm{E}_{\mathrm{v}}}{V_{m}}\right)^{1 / 2}=(C E D)^{1 / 2}
$$

where $V_{m}, \triangle \mathrm{E}_{\mathrm{v}}$, and $C E D$ are the molar volume, evaporation energy, and the cohesion energy density. The Hildebrand solubility parameter values are given in Table 1 .

Table 1 Solubility parameters of thermoplastic polymers

\begin{tabular}{ccc}
\hline Polymer & Solubility Parameter $\left(\mathbf{J} / \mathbf{c m}^{\mathbf{3}}\right)^{\mathbf{1 / 2}}$ & Solubility Parameter $\left(\mathbf{C} / \mathbf{c m}^{\mathbf{3}}\right)^{\mathbf{1 / 2}}$ \\
\hline PBI & 22.246 & 10.880 \\
PS & 19.030 & 9.308 \\
PES & 21.591 & 10.561 \\
PEI & 18.575 & 9.085 \\
\hline
\end{tabular}

Then, PES polymer was selected as the nanoprepreg matrix due to its close solubility parameter value to that of PBI. After the production of CNTs-PBI, nanoprepregs were fabricated by embedding them in PES films (GoodFellow, thickness $0.5 \mathrm{~mm}$ ) by the hot press after getting an insight into the interaction between the PBI and PES films. The formation by hot press was carried out for 30 minutes under a pressure of 10 bars at $320{ }^{\circ} \mathrm{C}$. The nanoprepreg

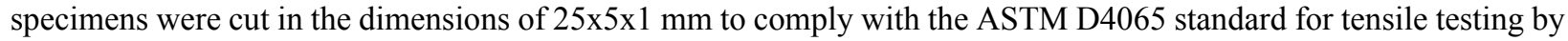
a dynamic mechanical analyzer. The produced nanoprepregs, which are two consecutive PES films, two PES films interlayered with a PBI layer in between, and two PES films interlayered with a CNTs-PBI layer were tested using TA Instruments, DMA Q800 under tension mode. The tests were performed at a frequency of $1 \mathrm{~Hz}$ over a temperature range of $25-250{ }^{\circ} \mathrm{C}$ with a ramp rate of $3{ }^{\circ} \mathrm{C} / \mathrm{min}$. The nanoprepreg specimens are illustrated in Fig. 2.

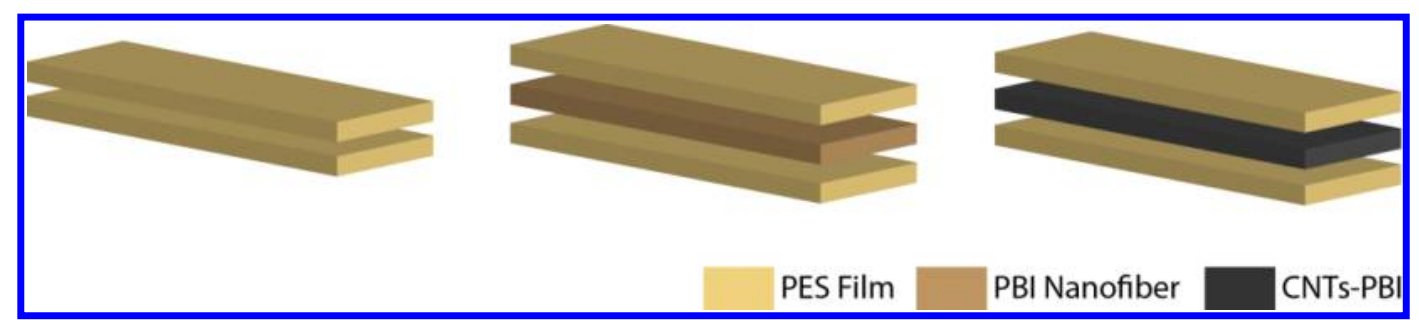

Fig. 2 An illustration depicting the fabrication of nanoprepreg specimens using PES film as matrix and PBI Nanofiber and CNTs-PBI Nanofiber as reinforcement

After the characterization of the nanoprepregs, laminated composite specimens were fabricated similarly to nanoprepreg fabrication process. The laminated composites consist of PES films and CNTs-PBI nanofiber interlayers where CF/PEEK prepregs (supplied from Mir AR-GE) were used as top and bottom layers. In each stacking sequence, nanoprepregs include a reference without an interlayer, and a neat PBI nanofibrous interlayer, and a CNTs-PBI interlayer to explore the effect of both neat and CNT-grown PBI interlayers. The stacking sequences of laminated composites with different interlayers are shown in Fig. 3.

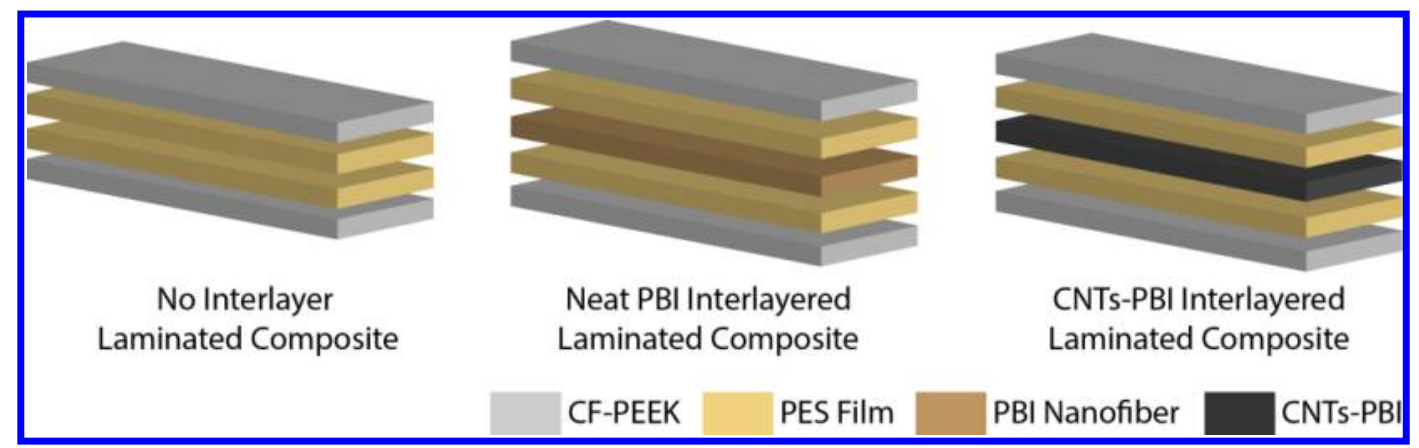

Fig. 3 The stacking sequences of the no interlayered, neat PBI interlayered, and CNTs-PBI interlayered CF/PEEK laminated composites 
According to the ASTM D790-17 standard, the composite specimens were manufactured with dimensions of $50 \times 10 \times 1.25 \mathrm{~mm}$. In order to generate reliable results, three specimens were manufactured for each configuration, resulting in 9 specimens. The average thicknesses of these specimens are shown in Table 2 , in which $0^{\circ}$ denotes the orientation of CF/PEEK prepregs. The flexural strength of the composite specimens was studied at room temperature by a Shimadzu AG-X Plus universal test machine with a load cell of $1 \mathrm{kN}$. The specimens were tested using a threepoint bending fixture with a test speed of $5 \mathrm{~mm} / \mathrm{min}$. An illustration of the flexural strength specimens and an actual manufactured specimen were shown in Fig. 4.

Table 2 Measured thicknesses of the laminated composite specimens for each configuration

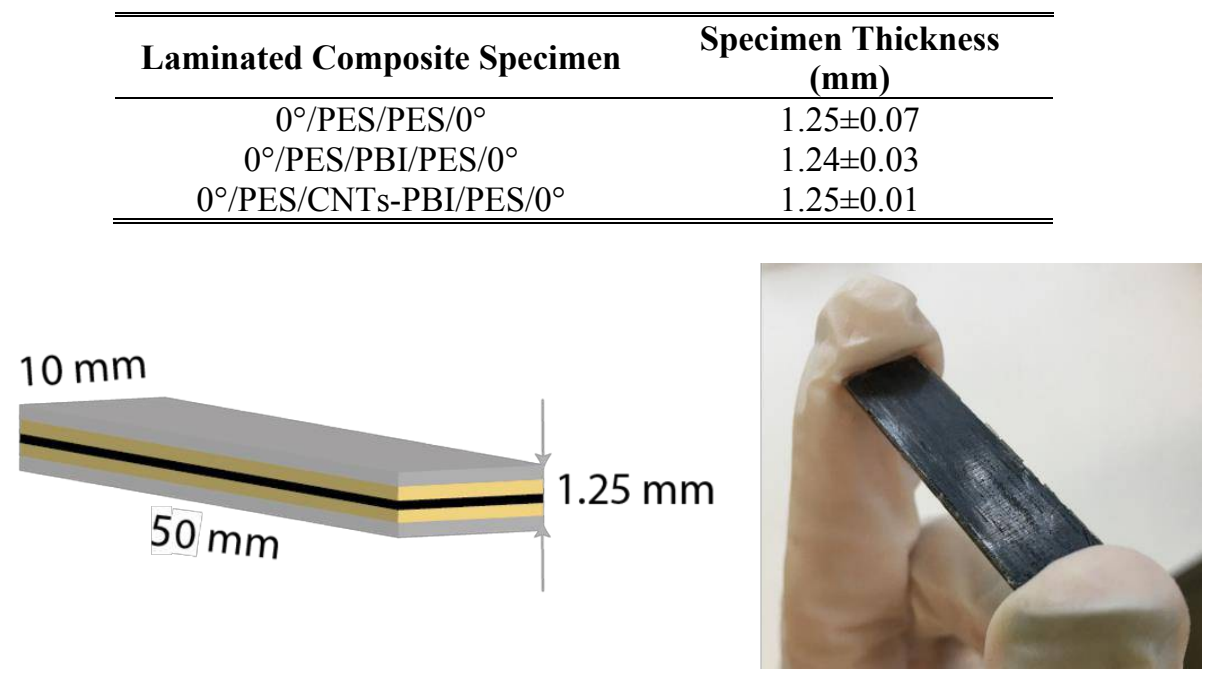

Fig. 4 (a) Illustration of the bending testing specimen, (b) Manufactured bending testing sample by hot press

\section{Results and Discussion}

\section{A. Morphological Analysis of CNTs-PBI interlayers}

Morphological characteristics of CNTs-PBI interlayers were investigated using two imaging techniques: SEM and TEM analyses. The images corresponding to SEM and TEM analyses are shown in Fig. 5, respectively. Fig. 5 (a) shows the uniform conformance and well-distribution of CNTs on PBI nanofibers. Fig. 5 (b) focuses on individual CNTs to determine the number of walls and wall thickness. The SEM image reveals that the grown CNTs are quite dense, relatively long, and radially aligned. The number of walls was found to be between 20 and 45 , while the outer diameter of the grown CNTs was $26.46 \pm 10.56 \mathrm{~nm}$.

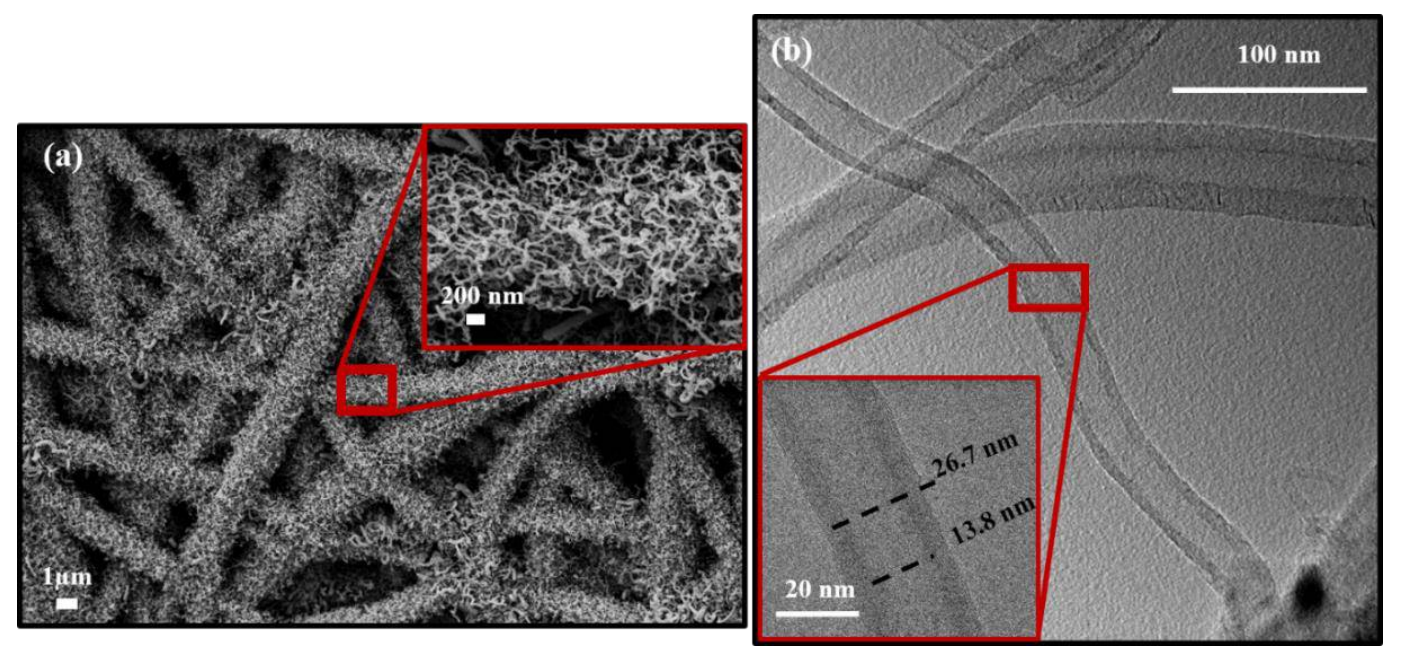

Fig. 5 (a) SEM image of the CNTs-PBI nanofibrous substrates, (b) TEM image of individual CNTs 
Raman spectroscopy results are presented in Fig. 6 in which two peaks, D and G, are quite evident. The D peak was detected at $1344 \mathrm{~cm}^{-1}$, results from the out-of-plane vibrations of $\mathrm{sp}^{3}$ atoms, which is an indication of the structural defects of carbon atoms while the $\mathrm{G}$ peak was located at $1593 \mathrm{~cm}^{-1}$, attributed to the in-plane vibrations of $\mathrm{sp}^{2}$ bonded atoms. The $\mathrm{I}_{\mathrm{g}} / \mathrm{I}_{\mathrm{d}}$ ratio was calculated as $1.08 \pm 0.04$, a similar result obtained by He et al. [24] with CVD-grown CNTs on micron-sized glass fibers. indicating defective CNTs resulted from low synthesis temperatures.

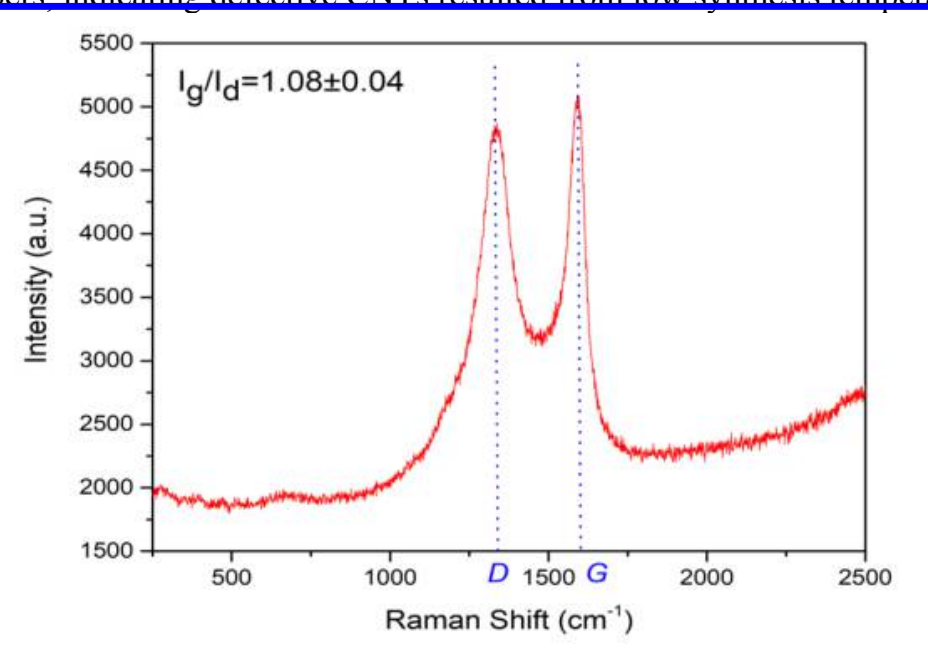

Fig. 6 Raman spectroscopy analysis of the CNTs-PBI nanofibers with nucleation and growth times of 15 and 10 mins, and catalyst molarity of $10 \mathrm{mM}$

\section{B. Thermo-dynamic Characterization of Nanoprepregs}

The thermo-dynamic characterization of the prepared nanoprepregs were investigated under tension to observe the effect of interlayering on the storage modulus. Fig. 7 presents the variation of storage moduli of neat PES film, PES/PBI/PES and PES/CNTs-PBI/PES nanoprepregs with increasing temperature. It was observed that reinforcing PES film with either a PBI or CNTs-PBI interlayer results in gradually shifted glass transition temperatures. This behavior indicates that compared to neat PES film, higher process temperatures are still sustainable with the integration of reinforcing interlayers. On the other hand, the decrease in the storage moduli in reinforced specimens was presumed to be resulting from process-related quality problems including air-trapned regions at the interface

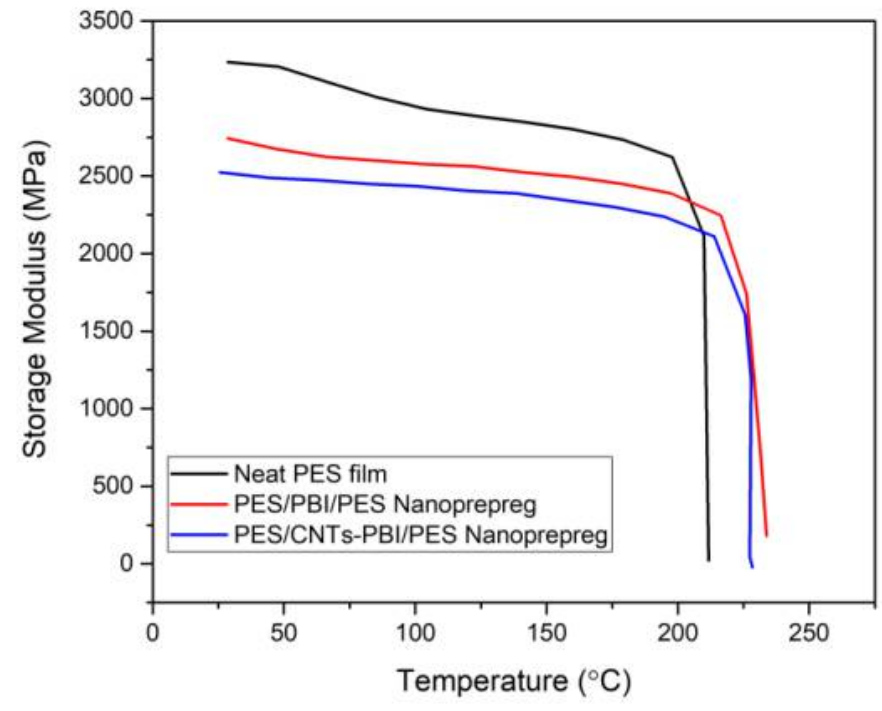

Fig. 7 Storage moduli of the nanoprepregs with respect to the temperature 


\section{Flexural Properties of reinforced CF/PEEK composites}

Flexural properties including flexural strength and modulus of the reinforced CF/PEEK composites were tested using a three-point bending fixture in order to investigate the improvements caused by the addition of interlayers. The flexural strength of the laminated CF/PEEK composite specimens without an interlayer, with PBI and CNTs-PBI interlayers is shown in Fig. 8.

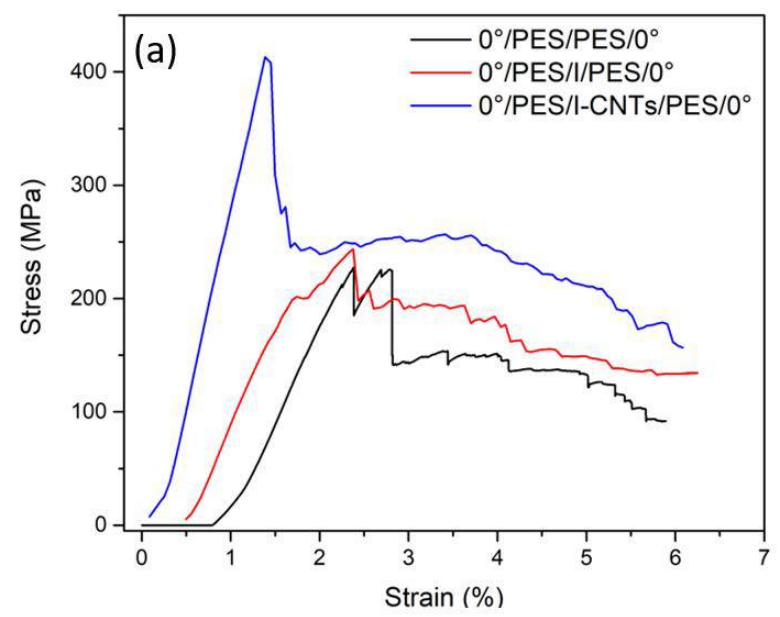

Fig. 8 Stress/Strain plot of laminated CF/PEEK composites

The results reveal that with the addition of either neat PBI or CNTs-PBI interlayers the flexural strength of the laminated composite structure was enhanced. This behavior indicates that the strategic placement of CNTs-PBI interlayers promoted good adhesion at the interface which could enable higher composite strength. On the other hand, even though addition of neat PBI interlayer only seems to be increasing the flexural strength but not affecting the flexural modulus noticeably, addition of CNTs-PBI interlayer enhances the modulus. The presence of CNTs promoted better adhesion to PES layers due to CNTs bridging effect compared to PBI nanofibrous interlayers. All the DMA and flexural tests are still being performed for further analysis. The post-test image of a laminated composite specimen is also given in Fig. 9.

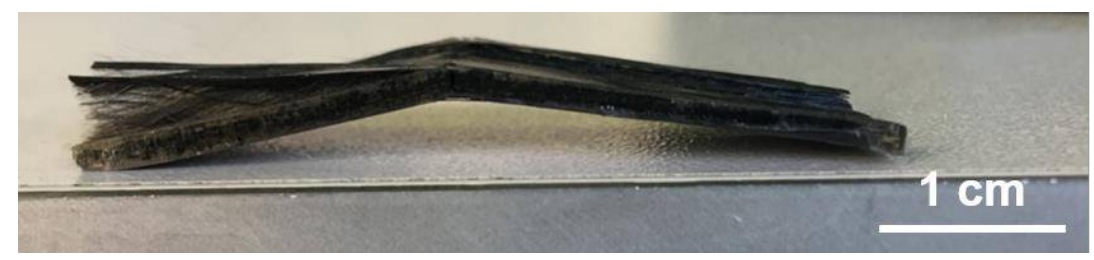

Fig. 9 Post-test image of a laminated composite specimen

\section{Conclusions and Future Work}

This study proposed a method that enables direct CNTs incorporation into thermoplastic matrices and also investigated these synthesized CNTs-PBI nanofibrous substrates as interleaves in laminated composite architectures. The morphological analysis of CNTs-PBI nanofibers showed that the uniform coverage and conformance of CNTs over PBI nanofibers achieved by the optimization of the innovative single-step CNTs synthesis. Dynamic mechanical analyses under tension mode demonstrated that glass transition temperatures were gradually enhanced with the addition of either PBI or CNTs-PBI interlayer, whereas no positive contribution to the storage modulus was observed. The impact of CNTs-PBI interlayers on the laminated CF-PEEK composites was tested under quasi-static flexural loading. Flexural strength and modulus of the composites were enhanced with the addition of neat PBI interlayers while significantly higher enhancements were observed with the CNTs-PBI interlayer. This behavior was attributed to better adhesion of the CNTs-PBI interlayer to adjacent layers due to CNTs bridging effect.

Strategic planning on reinforcing sequences along with a further optimization in manufacturing could create a drastic impact on reinforcing methods in thermoplastic composites. As future work, it is planned to expand the lamination strategy and number of tests conducted with genuinely produced CNTs-PBI interlayers by improving the manufacturing process of laminated composites, addressing different stacking sequences, and conducting dynamic mechanical analyses for comparison purposes. 


\section{Acknowledgments}

This study is funded by TUBITAK (The Scientific and Technological Research Council of Turkey) (Grant Number 116M427), under the R\&D Projects funding program. The authors would like to thank Firat Turgut for his assistance in CVD optimization and Dr. Özgür Duygulu for the efforts of TEM analyses.

\section{References}

[1] Coleman, J. N., Khan, U., and Gun'ko, Y. K. "Mechanical Reinforcement of Polymers Using Carbon Nanotubes." Advanced materials, Vol. 18, No. 6, 2006, pp. 689-706.

[2] Tjong, S. C. "Structural and Mechanical Properties of Polymer Nanocomposites." Materials Science and Engineering R: Reports, Vol. 53, No. 3-4, 2006, pp. 73-197. doi:10.1016/j.mser.2006.06.001.

[3] Khan, S. U., and Kim, J. K. "Improved Interlaminar Shear Properties of Multiscale Carbon Fiber Composites with Bucky Paper Interleaves Made from Carbon Nanofibers." Carbon, Vol. 50, No. 14, 2012, pp. 5265-5277. doi:10.1016/j.carbon.2012.07.011.

[4] Guzman de Villoria, R., Hallander, P., Ydrefors, L., Nordin, P., and Wardle, B. L. "In-Plane Strength Enhancement of Laminated Composites via Aligned Carbon Nanotube Interlaminar Reinforcement." Composites Science and Technology, Vol. 133, 2016, pp. 33-39. doi:10.1016/j.compscitech.2016.07.006.

[5] Bekyarova, E., Thostenson, E. T., Yu, A., Kim, H., Gao, J., Tang, J., Hahn, H. T., Chou, T. W., Itkis, M. E., and Haddon, R. C. "Multiscale Carbon Nanotube-Carbon Fiber Reinforcement for Advanced Epoxy Composites." Langmuir, Vol. 23, No. 7, 2007, pp. 3970-3974. doi:10.1021/la062743p.

[6] Garcia, E. J., Wardle, B. L., John Hart, A., and Yamamoto, N. "Fabrication and Multifunctional Properties of a Hybrid Laminate with Aligned Carbon Nanotubes Grown In Situ." Composites Science and Technology, Vol. 68, No. 9, 2008, pp. 2034-2041. doi:10.1016/j.compscitech.2008.02.028.

[7] Kim, S. W., Chung, W. S., Sohn, K. S., Son, C. Y., and Lee, S. "Improvement of Flexure Strength and Fracture Toughness in Alumina Matrix Composites Reinforced with Carbon Nanotubes.” Materials Science and Engineering A, Vol. 517, No. 1-2, 2009, pp. 293-299. doi:10.1016/j.msea.2009.04.035.

[8] Cho, M. "The Flexural and Tribological Behavior of Multi-Walled Carbon Nanotube-Reinforced Polyphenylene Sulfide Composites." Materials Transactions, Vol. 49, No. 12, 2008, pp. 2801-2807. doi:10.2320/matertrans.MRA2008262.

[9] Kim, M., Park, Y. Bin, Okoli, O. I., and Zhang, C. "Processing, Characterization, and Modeling of Carbon NanotubeReinforced Multiscale Composites." Composites Science and Technology, Vol. 69, No. 3-4, 2009, pp. $335-342$. doi:10.1016/j.compscitech.2008.10.019.

[10] Liang, J. Z., Zou, S. Y., and Du, Q. "Impact and Flexural Properties of Polypropylene Composites Reinforced with MultiWalled Carbon Nanotubes.” Polymer Testing, Vol. 70, No. July, 2018, pp. 434-440. doi:10.1016/j.polymertesting.2018.07.029.

[11] Zhou, Y., Pervin, F., Lewis, L., and Jeelani, S. "Fabrication and Characterization of Carbon/Epoxy Composites Mixed with Multi-Walled Carbon Nanotubes.” Materials Science and Engineering A, Vol. 475, No. 1-2, 2008, pp. $157-165$. doi:10.1016/j.msea.2007.04.043.

[12] Rathore, D. K., Prusty, R. K., Kumar, D. S., and Ray, B. C. "Mechanical Performance of CNT-Filled Glass Fiber/Epoxy Composite in in-Situ Elevated Temperature Environments Emphasizing the Role of CNT Content." Composites Part A: Applied Science and Manufacturing, Vol. 84, 2016, pp. 364-376. doi:10.1016/j.compositesa.2016.02.020.

[13] Allaoui, A., and El Bounia, N. "How Carbon Nanotubes Affect the Cure Kinetics and Glass Transition Temperature of Their Epoxy Composites? - A Review." Express Polymer Letters, Vol. 3, No. 9, 2009, pp. 588-594. doi:10.3144/expresspolymlett.2009.73.

[14] Díez-Pascual, A. M., Ashrafi, B., Naffakh, M., González-Domínguez, J. M., Johnston, A., Simard, B., Martínez, M. T., and Gómez-Fatou, M. A. "Influence of Carbon Nanotubes on the Thermal, Electrical and Mechanical Properties of Poly(Ether Ether Ketone)/Glass Fiber Laminates." Carbon, 2011. doi:10.1016/j.carbon.2011.03.011.

[15] Sandler, J., Werner, P., Shaffer, M. S. P., Demchuk, V., Altstädt, V., and Windle, A. H. "Carbon-Nanofibre-Reinforced Poly(Ether Ether Ketone) Composites." Composites Part A: Applied Science and Manufacturing, Vol. 33, No. 8, 2002, pp. 1033-1039. doi:10.1016/S1359-835X(02)00084-2.

[16] Zhang, L., Su, D., Jin, L., and Li, C. "Polyamide 6 Composites Reinforced with Glass Fibers Modified with Electrostatically Assembled Multiwall Carbon Nanotubes.” Journal of Materials Science, Vol. 47, No. 14, 2012, pp. 54465454. doi:10.1007/s10853-012-6434-y.

[17] Jin, Z., Pramoda, K. P., Xu, G., and Goh, S. H. "Dynamic Mechanical Behavior of Melt-Processed Multi-Walled Carbon Nanotube/PMMA Composites.” Chemical Physics Letters, Vol. 337, No. 1-3, 2001, pp. 43-47. doi:http://dx.doi.org/10.1016/S0009-2614(01)00186-5.

[18] Teng, C. C., Ma, C. C. M., Huang, Y. W., Yuen, S. M., Weng, C. C., Chen, C. H., and Su, S. F. "Effect of MWCNT Content on Rheological and Dynamic Mechanical Properties of Multiwalled Carbon Nanotube/Polypropylene Composites." Composites Part A: Applied Science and Manufacturing, Vol. 39, No. 12, 2008, pp. 1869-1875. doi:10.1016/j.compositesa.2008.09.004.

[19] Lv, P., Feng, Y. Y., Zhang, P., Chen, H. M., Zhao, N., and Feng, W. "Increasing the Interfacial Strength in Carbon Fiber/Epoxy Composites by Controlling the Orientation and Length of Carbon Nanotubes Grown on the Fibers." Carbon, Vol. 49, No. 14, 2011, pp. 4665-4673. doi:10.1016/j.carbon.2011.06.064. 
[20] Agnihotri, P., Basu, S., and Kar, K. K. "Effect of Carbon Nanotube Length and Density on the Properties of Carbon Nanotube-Coated Carbon Fiber/Polyester Composites." Carbon, Vol. 49, No. 9, 2011, pp. 3098-3106. doi:10.1016/j.carbon.2011.03.032.

[21] Bozali B., Yildiz K., Köken D., Zakaria A., Cebeci F., Cebeci H., O.-Y. E. "Anchoring Radially Oriented Carbon Nanotubes with Polybenzimidazole (PBI) Nanofibers.” Advanced Materials Technologies, Vol. Submitted, 2019.

[22] Bozali, B. Production of Carbon Nanotube Reinforced Nanoprepregs And Their Characterization. Istanbul Technical University, 2018.

[23] Ozden-Yenigun, E., Atilgan, C., and Elliott, J. A. "Multi-Scale Modelling of Carbon Nanotube Reinforced Crosslinked Interfaces." Computational Materials Science, Vol. 129, 2017, pp. 279-289. doi:10.1016/j.commatsci.2016.12.019.

[24] He, D., Fan, B., Zhao, H., Lu, X., Yang, M., Liu, Y., and Bai, J. "Design of Electrically Conductive Structural Composites by Modulating Aligned CVD-Grown Carbon Nanotube Length on Glass Fibers." ACS Applied Materials and Interfaces, Vol. 9, No. 3, 2017, pp. 2948-2958. doi:10.1021/acsami.6b13397. 